\title{
Dankesrede anlässlich der Preisverleihung
}

\author{
Robert Pfaller (Linz)
}

\section{Sehr geehrte Damen und Herren,}

meine Freude über diesen Preis des Psychoanalytischen Seminars Zürich ist außerordentlich groß. Ich freue mich nicht allein, diese Anerkennung für meine wissenschaftliche Arbeit zu erhalten; es freut mich vor allem, diesen Preis von ihnen verliehen zu bekommen. Ein kluger Wiener Kollege unter den Kulturwissenschaftlern hat mir gratuliert und gesagt: «Das ist überhaupt der beste Preis, den Du gewinnen kannst.» Das sehe ich auch so.

Denn das Psychoanalytische Seminar Zürich ist in der Welt berühmt als ein Paradebeispiel unter den - leider nicht allzu zahlreichen - psychoanalytischen Institutionen, die ihre Wissenschaft nicht bloß als klinische Disziplin mit einigen durchaus entbehrlichen kulturbezogenen Anhängseln, sondern grundsätzlich als eine Verbindung von klinischen Problemen und Fragen der Kunst, der Kultur und der Gesellschaft begreifen. Sigmund Freud selbst, der zum Beispiel über Zwangshandlungen nicht nachdenken konnte, ohne Fragen der Religionen und ihrer Geschichte zu stellen, und der die Analyse des Ich nur auf dem Weg über die Massenpsychologie sowie über die Frage nach dem Unbehagen in der Kultur durchführen konnte, hat diese wechselseitige Abhängigkeit der psychoanalytischen Gegenstandsbereiche ja deutlich vorgeführt.

Psychoanalyse so aufzufassen heißt, erkannt haben, dass sich die klinischen Fragen nicht klären lassen, wenn man nicht zugleich die gesellschaftlichen Verhältnisse einer Befragung unterzieht - und umgekehrt. Und es bedeutet auch wenn ich die Worte aus einem großartigen Buchtitel eines Ihrer Gründer, Paul Parin, zitieren darf: «Es ist Krieg, und wir gehen hin.» Die Psychoanalyse begibt sich unter solchen Voraussetzungen eben dorthin, wo die Konflikte dieser Welt und dieser psychischen Apparate wirklich stattfinden; wo die Erkenntnishindernisse, mit denen sie als Wissenschaft brechen muss, zugleich Ideologien sind, ohne die die Gesellschaft nicht so funktionieren könnte, wie sie es tut.

Die Psychoanalyse ist, wie der Philosoph Louis Althusser bemerkt hat, eine konfliktuelleWissenschaft. Das bedeutet: wenn sie sich den Widerständen widmet, die sich ihren eigenen Erkenntnisvorgängen in den Weg stellen, dann bekommt sie es nicht nur mit epistemologischen Problemen zu tun, sondern mit leidenschaftlich verteidigten Gewissheiten eines systemdienlichen ideologischen common sense; 
und sie stellt dann jene Fragen, deren Antworten sich wirklich zu erkennen lohnt. Dass man es sich mit einem so großen Anspruch nicht leicht macht, ist klar - aber andererseits: Mit jedem kleineren Anspruch macht man es sich ja von vornherein unmöglich, auch nur diesen einzulösen.

In diesem Anspruch, der übrigens auch von den beiden Wiener Gruppen, denen ich angehöre, der Neuen Wiener Gruppe Lacan-Schule sowie der Forschungsgruppe Psychoanalyse «stuzzicadenti», geteilt wird, fühle ich mich Ihnen verbunden. Das erklärt einen großen Teil meiner Freude.

Ein weiterer Teil meiner Freude rührt daher, dass es großartig ist, wenn diejenigen, deren Instrumente man entlehnt hat, um etwas Ungewöhnliches damit anzustellen, dies als wertvollen Beitrag zur Verbesserung und weiteren Schärfung ihrer Instrumente wahrnehmen. An diesem Punkt verbindet sich meine Freude mit Erleichterung: Denn indem ich einige begriffliche Netze aus der Psychoanalyse mit solchen aus Philosophie, Kulturwissenschaft und Kunst verbunden habe, ist es mir, wie mir scheint, gelungen, einen Fisch an Land zu ziehen, der für meinen begrenzten individuellen Forscherappetit viel zu groß - und überdies mit möglicherweise dem einen oder anderen Schatz in seinem Bauch versehen und insofern ein wenig unheimlich - ist. Ihr Interesse an meinen Ergebnissen lässt mich hoffen, dass Sie mich mit diesem Fang nicht alleine lassen und zusammen mit Ihren geborgten Netzen auch einige saftige Teile dieses Riesentieres zum Genuss sowie zur weiteren Untersuchung an sich nehmen werden.

Wie Sie wissen, betrifft meine - vor allem auf die Vorstöße des Psychoanalytikers Octave Mannoni gestützte - Entdeckung den Umstand, dass es nicht nur die bekannten Einbildungen mit bekennenden Eigentümern, sondern daneben auch noch ganz andere, Einbildungen ohne Eigentümer, gibt. Wir pflegen nicht nur die Überzeugungen, mit denen wir uns voll und ganz identifizieren und aus deren Besitz wir Selbstachtung beziehen. Ebensosehr bestimmen uns Einbildungen, die wir als blöd oder unsinnig von uns weisen, die aber dennoch beträchtliche Macht über unsere Handlungen ausüben können - wie das Horoskop oder der Sportbericht, die wir eigenartigerweise oft noch vor dem politischen Teil der Tageszeitungen lesen müssen, oder ebenso auch die Möglichkeit, den streikenden Computer zu bestrafen oder mit dem eigenen Auto zu sprechen, wenn es nicht starten will.

Solche immer schon durchschaute Illusionen ermöglichen unseren Genuss an der Kunst: Zum Beispiel, wenn bei einem Gemälde der trompe-l'oeil-Malerei es so scheint, als ob sich vor einer Porträtdarstellung noch eine geborstene Glasplatte befände. «Wie fein, man hätte glauben können, hier wäre echtes Glas 
zerbrochen ...», sagt man dann zum Beispiel, und hat es doch nie geglaubt. Der ästhetische Genuss jedenfalls ist hier nur denjenigen zugänglich, die die Illusion durchschauen. Wenn es jemanden gäbe, der sie nicht durchschaut und der das bloß für einen Transportschaden hält, dann hätte diese Person jedenfalls keinen Genuss. Dies muss insbesondere für Philosophen eine Lehre sein: Denn gemeinhin wird in meiner Branche gern angenommen, dass das Glück nur denjenigen zugänglich wäre, die ganz in einer Illusion gefangen sind; und dass der leiseste Anflug von Erkenntnis oder Bewusstsein bereits der Anfang vom Verlust dieses Glücks wäre. Beim trompe-l'oeil jedoch ist es genau umgekehrt: Der Genuss ist nur den Hellsichtigen und Ungetäuschten zugänglich.

Solche aufgehobenen Illusionen sind als Glücksprinzip wirksam in allem, was uns im Leben Freude macht: in der exzessiven Begeisterung des Spiels; in den alltäglichen Fiktionen der Höflichkeit und der Eleganz, zum Beispiel in der mondänen Dimension der Tabakkultur; und auch in den kleinen Anhänglichkeiten, die wir gegenüber bestimmten Kultobjekten im Alltag pflegen - zum Beispiel LieblingsCDs, Fußballklubs, gewissen Stofftieren, sentimental favorisierten Autotypen oder Filmstars, den Reliquien der Liebe sowie den sogenannten «Kultserien» im Fernsehen.

Für die Philosophie ergab sich aus diesen Funden die Erkenntnis, dass das, was wir gemeinhin für einen Aufklärungsprozess halten, in Wahrheit nur ein Prozess asketischerVerinnerlichung ist: Die Preisgabe kleinerVerrücktheiten, ritueller Praktiken und kultischer Objekte verdankt sich keinem Erkenntnisgewinn. Denn das «bessere Wissen», dass das alles Unsinn ist, musste bereits vorher vorhanden gewesen sein; anders ließe sich nicht erklären, weshalb all das einmal mit Lustgewinn gepflegt wurde. Aufklärung heißt nur, dass dieses bessere Wissen nun plötzlich als Argument dafür genommen wird, eine totale Abstinenz gegenüber solchen lustigen Verrücktheiten zu pflegen. Nicht die Rationalität des Objekts, sondern seine Eignung zur totalen Identifizierung ist das Kriterium der sogenannten Aufklärung. Und wir haben nicht an Erkenntnis dazu gewonnen; vielmehr haben wir begonnen, uns selbst ernster zu nehmen und nur noch jene Einbildungen an uns zu dulden und wahrzunehmen, mit denen wir uns ganz identifizieren können. Psychoanalytisch gesprochen: Wir sind nicht klüger geworden, sondern nur humorloser. Und wir haben nicht aufgehört, an Einbildungen zu glauben; im Gegenteil: An die Stelle aufgehobener Illusionen haben wir immer festere, eigene Überzeugungen gesetzt. Die Idee von den goldenen Zeiten, in denen sich die Menschen in ihren Illusionen geborgen fühlten, während wir Modernen in skeptischer, «transzendentaler Obdachlosigkeit» herumirrten, ist ein Mythos. Früher hat 
man weniger geglaubt; wir hingegen sind die, die wirklich an etwas glauben - und nichts anderes dulden als das, woran wir ganz und fest glauben können.

Für die psychoanalytische Theorie ergibt sich aus diesen Erkenntnissen, wie ich glaube, ein massiver Bedarf des Überdenkens von lange tradierten Grundannahmen. Angesichts der Alltäglichkeit dieser Normalgestalten der Verleugnung wird man zum Beispiel eine präzisere Bestimmung der Perversion entwickeln müssen: Man wird zu klären haben, unter welchen Bedingungen man es mit der Verleugnung als allgemeinem kulturellem Lustprinzip zu tun hat, und welche Zusatzbedingungen auftreten müssen, damit die Verleugnung zu jenem spezifischen Abwehrmechanismus wird, der die Perversion bedingt.

Auch die traditionelle, von Freud selbst mitunter - etwa in «Totem und Tabu» - vorgeführte Verachtung psychoanalytischer Theorie gegenüber den kulturellen Praktiken der Magie erscheint mir vor diesem Hintergrund unhaltbar. Die Magie ist kein Resultat einer infantilen, narzisstischen Weltauffassung, worin zwischen Wünschen und Herstellen noch kein Unterschied getroffen werden kann. Vielmehr wird man sich zu jeglichem psychoanalytischen Verständnis der Magie verstärkt auf die bahnbrechende Erkenntnis Freuds aus dem Fetischismus-Aufsatz stützen müssen: Nämlich dass die fetischistischen Praktiken das Ergebnis der Aufgabe einer Illusion sind. Nur wer eine infantile Illusion überwunden hat, kann Freude aus dem Umgang mit einem Fetisch gewinnen. Wer hingegen noch in der Illusion steckt, der braucht gar keinen Fetisch.

Eine solche Herangehensweise wird es ermöglichen, nicht nur die kleinen Magien des zivilisierten Alltagslebens wahrzunehmen und zu würdigen, sondern auch den von Freud selbst vorgeführten Respekt gegenüber der klassischen antiken Kultur sowie den heidnischen Kulturen insgesamt wiederzugewinnen. Die dort beobachtbare Freude an der Figurativität und die grundsätzliche Überzeugung von der Diesseitigkeit und Darstellbarkeit des Göttlichen wird uns dann nicht mehr als narzisstische Naivität erscheinen, sondern gerade als deren Gegenteil. Denn nicht durch Naivität werden Dinge und Figuren uns zu zärtlich gehüteten Kultobjekten und Fetischen, sondern nur durch das viel paradoxere und tückischere Prinzip der aufgehobenen Illusion. (Um ein berühmtes, aktuelles Beispiel für die Verachtung der heidnischen Kulturen zu nennen: So treffend und bedeutsam die grundlegenden Analysen von Béla Grunberger und Pierre Dessuant in bezug auf das narzisstische Prinzip des Christentums sind, so sehr verfallen die Autoren in eine simple Auffassung der antiken Kultur. Der Narzissmus, den sie dieser zuschreiben, lässt sich dann nicht einmal mehr gut von jenem anderen - und so verschiedenen - 
trennen, den sie zurecht und mit entscheidenden politischen Konsequenzen am Christentum diagnostizieren.)

Weil die psychoanalytische Theorie aber - wie gesagt - die Besonderheit hat, sich nicht selbst kritisieren zu können, ohne zugleich etwas am Zustand der Welt zu kritisieren, möchte ich auch andeuten, an welchem Punkt der Welt diese Kritik heute auftreffen muss. Wie der Soziologe Richard Sennett - übrigens mit großer psychoanalytischer Schärfe - erkannt hat, leben wir in einer sich zunehmend narzisstisch gestaltenden Kultur. Alle Vorgänge werden zunehmend der Frage unterworfen: «Was hat das mit mir zu tun?» Eine den neoliberalen Entwicklungen besonders dienliche Ideologie bringt die Individuen heute schon reflexartig und automatisch dazu, ständig ganz sie selbst sein zu wollen. «Be yourself!» - diese Devise hallt gegenwärtig nicht nur im HipHop permanent als Refrain wider.

Diese Sehnsucht nach dem eigenen Selbst und nichts sonst stellt aber, wie Sie leicht sehen können, einen massiven Defekt der Objektlibido dar. Was für ein Unterschied gegenüber einer Popkultur, die noch sagen konnte «We want the world, and we want it now!»

Das kulturell induzierte Interesse am eigenen Selbst führt heute dazu, dass Individuen sogar noch mit dem Gefühl der Befreiung heute diejenigen Güter von sich aus ablehnen, die man ihnen ohnehin entziehen will. Die narzisstische Ablehnung von Äußerlichkeiten wie z. B. Höflichkeits- oder Eleganz-Formen, führt zu asketischer Verinnerlichung und in der Folge zu politischem Verzicht.

Man darf und soll immer ganz man selbst sein - das heißt heute zum Beispiel: Man darf ins Fernsehen, wenn man dort ganz «authentisch» jenen Freak spielt, der man angeblich privat ist; aber man hat keinen Anspruch mehr darauf, mit dem Fernsehen relevante Öffentlichkeiten zu erreichen und Fragen von gesellschaftlichem Interesse zu erörtern. Wir beobachten eine Entpolitisierung des öffentlichen Raumes, die ermöglicht wird durch eine totale Unterwerfung dieses öffentlichen Raumes unter private Ansprüche.

Eine solche Beraubung der Individuen lässt sich wahrscheinlich gar nicht wirksam bewerkstelligen, wenn man sie ihnen nicht als Befreiung, als Verwirklichung ihrer ureigensten Interessen verkauft. Darum gibt sich unsere Kultur gern als permissiv und relativistisch aus. Ihr kategorischer Imperativ lautet: «Du darfst alles, wenn Du es nur selbst ganz willst.»

Das Studium der Einbildungen ohne Eigentümer lehrt uns jedoch, dass Individuen ihre größten Glücksmöglichkeiten dort vorfinden, wo sie eben nicht ganz sie selbst sind; wo sie vielmehr ein wenig gespalten sind und - entsprechend 
der Formel von Mannoni - sagen können «Ich weiß zwar, dass das Blödsinn ist, dennoch aber ist es großartig!»

Nichts ist darum repressiver als eine Gesellschaft, welche die Legitimität von Ansprüchen von deren Ich-Konformität abhängig macht. Wenn man nur das darf, was man selbst ganz will, und nichts sonst, dann darf man darum in Wahrheit sehr, sehr wenig. Das ist der Grund für die extremen asketischen Tendenzen in unserer Kultur - für den von Slavoj Zizek treffend erkannten «Non-ism», der uns entweder Bier ohne Alkohol, Sex ohne Körper, Kaffee ohne Koffein, Schlagsahne ohne Fett, oder aber totale Rauchverbote, politisch korrekte Schimpfverbote oder auch Kunstbeseitigungen im öffentlichen Raum einbringt.

Wenn der Begriff der Sublimierung ein Begriff ist - und nicht nur der Name eines in der psychoanalytischen Theorie ungelösten Problems - wenn dieser Begriff also einen theoretischen Sinn hat, dann diesen: Dass es die Aufgabe der Kultur ist, den Individuen dasjenige genießbar zu machen, was sie selbst an sich niemals zur vollständigen Identifizierung bringen können. Denn kein Raucher zum Beispiel hat jemals ein völlig ungebrochenes, durchwegs positives Verhältnis zu seiner Droge. Und niemand hat das Rauchen jemals für ungefährlich gehalten. Genau darum aber - weil es zwiespältig und gefährlich ist - kann es, wie Richard Klein richtig bemerkt hat, unter feierlichen kulturellen Bedingungen als etwas Großartiges, Sublimes erfahren werden.

Erwachsensein heißt nach psychoanalytischer Auffassung übrigens genau dies: zum Sublimen fähig sein. Das heißt: Nicht nur das unproblematisch Lustvolle sowie das schlichtweg Unlustvolle zu kennen, sondern auch das problematisch Lustvolle, das sich nur unter Zuhilfenahme kultureller Mittel in etwas Triumphales verwandeln und genießen lässt. Die neoliberale Ideologie des «Be Yourself!» hingegen arbeitet daran, die Welt nur noch in eine problemlos lustvolle und eine schlichtweg unlustvolle Hälfte zu spalten. Mit anderen Worten, diese Ideologie erzieht uns zu gehorsamen Bewohnern einer infantilen Welt.

Die Psychoanalyse zeigt uns, wie ich glaube, sehr klar den Standpunkt an, den wir, ob wir wollen oder nicht, hier einnehmen müssen, und sie gibt uns die theoretischen Waffen in die Hand, um ihn zu vertreten. Ich würde sagen, hier ist heute Krieg, und ich meine: hier müssen wir hingehen. Ich danke Ihnen. 